\title{
Screening The Efficacy of Melatonin On Neurodegeneration Mediated By ER Stress, Inflammation And Oxidative Damage
}

Fathima Hajee Basha

BS Abdur Rahman University: B S Abdur Rahman Crescent Institute of Science \& Technology

S. Hemalatha ( $\nabla$ hemalatha.sls@bsauniv.ac.in)

B S Abdur Rahman Crescent Institute of Science \& Technology https://orcid.org/0000-0002-8150-7721

\section{Research Article}

Keywords: Neurodegeneration, ER stress, Melatonin, Inflammation, Antioxidant, in silico

Posted Date: August 24th, 2021

DOI: https://doi.org/10.21203/rs.3.rs-769141/v1

License: () (7) This work is licensed under a Creative Commons Attribution 4.0 International License. Read Full License 


\section{Abstract}

Neurodegeneration may be defined as a clinical condition wherein neurons gradually lose their structural integrity, viability, functional abilities and the damage inflicted upon the neurons is often irreversible. The number of elderly patients suffering from Neurodegenerative disorders is expected to rise tremendously over the next couple of years. Thus, there is an urgent need to delve into and study the underlying cause and mechanisms, so that we may be able to develop more effective therapeutic strategies and drugs and better understand the origin and progression of the disease.The various mechanisms that have been observed to contribute to neurodegeneration include aggregation and accumulation of misfolded proteins, impaired autophagy, oxidative damage, neuroinflammation, mitochondrial defects, increased SUMOylation of proteins, impaired UPR pathways, disruption of axonal transport.Melatonin, a neurohormone is involved in a variety of functions including scavenging free radicals, synchronizing the circadian rhythm, mitigating immune response. Melatonin has shown to modulate the UPR pathway, antioxidant pathway through Nrf2 and inflammatory pathway through NFKB. The study aims to determine the efficacy of melatonin on neurodegeneration mediated by ER stress, inflammation and oxidative damage through in silico approaches. The molecular targets chosen were ATF6, XBP1, PERK, Nrf2, NFKB and they were docked against melatonin. Additionally various physiochemical analysis such as ADME were also carried out to determine its drug ability. The findings were that melatonin not only shows excellent interactions with the targets but also possess drug-like physicochemical properties that makes it a valuable choice for the treatment of neurodegenerative disorders.

\section{Introduction}

Neurodegeneration may be defined as a clinical condition wherein neurons gradually lose their structural integrity, viability, functional abilities and the damage inflicted upon the neurons is often irreversible. The number of elderly patients suffering from Neurodegenerative disorders is expected to rise tremendously over the next couple of years. [1]. While current treatment strategies only focus on alleviating the symptoms, there is not much it contributes to stop the progression of the disease and they are often accompanied by devastating side effects. Thus, there is an urgent need to delve into and study the underlying cause and mechanisms, so that we may be able to develop more effective therapeutic strategies and drugs and better understand the origin and progression of the disease.[2].

The initial step towards developing better therapeutic strategy is to determine the molecular mechanisms for the same. The various mechanismsthat have been observed to contribute to neurodegeneration include aggregation and accumulation of misfolded proteins, impaired autophagy, oxidative damage, neuroinflammation, mitochondrial defects, increased SUMOylation of proteins, impaired UPR pathways, disruption of axonal transport. In this paper we shall only discuss UPR, neuroinflammation and oxidative damage in depth.[3, 4].

Melatonin is a neurohormone that is secreted by the pineal gland of the brain. This hormone is secreted in response to darkness, which is why the melatonin concentration is found to ten times higher at night than during daytime.[4, 5].But as we age, the melatonin levels also drop simultaneously. A myriad of diseases like neurodegenerative disorders, cancers show deregulation of melatonin, which points out to the fact the melatonin regulation is crucial in order to be protected from various kinds of diseases. What makes it an excellent choice for a therapeutic drug for treating neurodegenerative disorders is its ability to penetrate the blood brain barrier, an ability confined to small number of molecules.[6].

The brain consumes about $20 \%$ of the body's oxygen and this great level of consumption of oxygen leads to oxidative stress. Oxidative stress is wherein there is an increased production of chemical entities called as Reactive Oxygen Species (ROS). Increased ROS in the neuronal cells pose a threat of altered gene expression, damage the genetic material \& biomolecules and ultimately decrease the cell viability of neuronal cells. Moreover, brain cells have a higher proportion of fatty acids, which make them even more susceptible to oxidative damage this. To counteract these ROS, our body possesses certain systems called as Antioxidant systems, which scavenge those free radicals / ROS. One such extremely potent antioxidant is the neurohormone melatonin.[5].Melatonin exerts its effects by binding its receptors MT1 and MT2, which are essentially G-Protein coupled Receptors. While MT1 is found abundantly in the CNS, MT2 is confined to a certain areas such as the hippocampus of the brain. Melatonin is capable of directly blocking oxidative stress, increasing the expression of antioxidant enzymes like Glutathione peroxidase, catalase, etc.[7]

One specific signalling pathway melatonin is known to modulate is the Nrf2 antioxidant signalling pathway. Melatonin not only exerts neuroprotective effects but also a range of other effects like hepatoprotective, nephroprotective and cardioprotective effects through Nrf2 signaling.Nuclear factor erythroid 2-related factor 2 (Nrf2) is the major sensor of oxidative stress occurring in the cells and its major aim is to restore the redox balance. In normal conditions the Nrf2 peptide is kept translationally inactive by a protein termed as Kelch-like ECH-associated protein 1 (Keap1). Upon conditions of oxidative stress the dissociation of Keap1 and Nrf2 is facilitated, due to which Nrf2 is translocated to the nucleus and it later activates the genes controlling antioxidant defences such as Superoxide dismutase (SOD), Catalase (CAT) ,etc[8, 9].

Melatonin also modulates the UPR pathways such that there is reduced UPR induced apoptosis and increase in the neuronal viability.[6] Most of the neurodegenerative disorders, though different in their clinical symptoms, share few common pathogenic features including protein misfolding, protein aggregation, etc $[10,11]$. Thus, this narrows down our focus to the cellular organelle that is concerned with the quality control and homeostasis of proteins, i.e. the Endoplasmic Reticulum(ER).ER is the cell organelle wherein protein synthesis, folding and transport occurs.[12]. Under normal conditions, ER resident chaperones ensure correct protein folding and facilitate the degradation of misfolded proteins via various pathways. However, recurrent misfolding of proteins can lead to their accumulation within the ER and this generates ER stress. These occurrences collectively elicit the Unfolded Protein Response (UPR)[13, 14].

UPR can be initiated through three transmembrane proteins namely IRE1, ATF6 and PERK. All the aforementioned proteins possess stress-sensing domains. Thus, when there is an episode of protein accumulation or protein misfolding, either of the three proteins get activated. If IRE1 is activated, it eliminates an intronic sequence from another protein called as XBP1. After which XBP1 is translocated to the nucleus and there it activates the UPR genes. If ATF6 is activated ,it is translocated to the Golgi Apparatus. After translocation it is cleaved by two endopeptidases namely S1P and S2P. This cleavage results in the release of a fragment called as ATF6f, which is now translocated to the nucleus so that it activates the UPR genes. Lastly, if PERK is activated it 
phosphorylates a protein called as elF2a. elF2a facilitates the translation of a protein termed as ATF4 which is later translocated to the nucleus so that it can activate the UPR genes. The consequence of activating UPR is to either promote protein folding or promote ER associated degradation (ERAD)[15-17].

ER stress and UPR are considered as both hallmarks and biomarkers for many neurodegenerative disorders. UPR activators such as ATF6 are also targeted for the treatment of cancers such as gliomas.[18]For instance, in AD patients, an increased level of phosphorylated PERK and IRE1a are found alongside phosphorylated Tau protein, which is a hallmark of the disease.[19]

Melatonin most importantly also helps in reducing $A \beta$ induced toxicity in AD which is a major pathological hallmark of the same. Besides it also helps in preventing the formation and aggregation of $A \beta$ altogether.[20,21]. Similarly, it helps in reducing a-synuclein induced toxicity and $a-s y n u c l e i n$ aggregation which is a major pathological hallmark of PD.[5].

The next major protective effect is the anti-inflammatory effect of melatonin. An increased state of neuroinflammation is also observed in a number of neurodegenerative disorders. This inflammatory environment is also thought to lead to the progression of these diseases. Melatonin is shown to prevent the activation of microglial cells, astrocytes. Besides, it is also capable of inhibiting NFKB which in turn leads to the inhibition of production of various proinflammatory cytokines such as IL-6, TNF-a[20].Nuclear Factor kappa-light-chain-enhancer of activated B cells (NFKB) is a pivotal transcription factor for innumerable pro-inflammatory cytokines and chemokines. Itis also crucial in maintaining the homeostasis of neuronal and glial cells. Under normal conditions it is present as a complex of 2 subunits namely p50 and p65 and an inhibitory subunit namely lkB. Upon appropriate stimuli, the lkB is degraded and the remaining complex is translocated to the nucleus. After reaching the nucleus, it regulates the gene expression. Besides, there is also evidences of crosstalk between Nrf2 signalling and NFKB as some NFKB binding sites are observed in the promotor region of the Nrf2 gene. Nrf2 protein is able to inhibit the activity of NFKB and thus this can decrease the inflammatory state in a disease scenario.[8, 22, 23]

\section{Experimental Methods And Softwares Used}

\section{Protein preparation}

The PDB structures for ATF6 (PDB ID: -2DGC),PERK (PDB ID: -5SV7),XBP1 (PDB ID: -6IAK), Nrf2 (PDB ID:- 2FLU), NFKB (PDB ID:- 1SVC) were retrieved from the PDB web server (https://www.rcsb.org/). Macromolecule preparation was done by deleting water molecules, adding polar hydrogens and adding Kollman charges. The presence of any missing residues were determined using the misc option and the repair missing atoms function was used to repair the structure. Any undesirable ligand, chemical entities and protein chains were deleted as per the description of the structure in PDB in order to reduce the computational resource and space.

\section{Ligand preparation}

The SDF format for melatonin (PubChem ID-896)was retrieved from the PubChem server .The SDF format was converted to PDBQT format using OpenBabel. The ligand preparation was done by computing and adding Gasteiger charges.

\section{Molecular Docking}

Autodock software at default settings was used for the docking and for obtaining the binding energies. The docking done was blind as there was no adequate knowledge about the binding site of the macromolecule. The spacing between grid points was adjusted as 1.000 . The $X, Y, Z$ values of the grid box were set accordingly. The output form chosen was Lamarckian Genetic Algorithm and the number of runs per molecule was set as the default (10 runs). The proteinligand complex was written for every molecule using Autodock's write complex function.

\section{D protein ligand interaction profiling}

The PDB format of those complexes was later uploaded on the PoseView tool of the protein plus server (https://proteins.plus/) for the 2D protein-ligand interaction profiling so as to gain a deeper insight into the interaction between the ligand and the amino acids of the protein.

\section{Lipinski screening}

The ligands were analyzed for the Lipinski rule of 5 using the http://www.scfbio-iitd.res.in/software/drugdesign/lipinski.jsp server. The SDF format of melatonin was uploaded in the input section of the server and the $\mathrm{pH}$ was set as 7 (default). This is to analyze the drug-likeness of the molecule on the basis of the five Lipinski rules.

\section{ADME analysis}

The ligands were analyzed for their ADME properties using the Swiss ADME server.The SMILES format for melatonin was retrieved from the Pubchem server and entered in the input box of the server. This is to analyze the physicochemical descriptors (such as BBB permeability), of the ligand molecule.

\section{Analysis of molecular properties and prediction of bioactivity}

Lastly, melatonin was analyzed for its molecular properties and bioactivity using the molinspiration server (https://www.molinspiration.com/). The SMILES format of melatonin was retrieved from the PubChem server and this served as an input for calculating molecular properties followed by the prediction of the bioactivity score.

\section{Results And Discussion}




\section{Molecular Docking}

Molecular Docking is crucial component of Drug Discovery and development process. It helps in determining the best ligand position and is often used in combination with various other techniques in order to obtain useful information and for the better analysis of lead compounds.[24, 25]. The above energies signify the versatility of the neurohormone melatonin as it is involved in a myriad of cellular processes and functions (Table 1)

Table 1 -shows the binding energies (in kJ) of melatonin with ER arm proteins, antioxidant signaling protein Nrf2 and the inflammatory signaling protein NFKB

\begin{tabular}{|c|c|c|c|c|c|}
\hline $\begin{array}{l}\text { Macromolecule } \rightarrow \\
\text { Ligand }\end{array}$ & $\begin{array}{c}\text { ATF6 } \\
\text { (Binding } \\
\text { energy in kJ) }\end{array}$ & $\begin{array}{c}\text { PERK } \\
\text { (Binding } \\
\text { energy in kJ) }\end{array}$ & $\begin{array}{c}\text { XBP1 } \\
\text { (Binding } \\
\text { energy in kJ) }\end{array}$ & $\begin{array}{c}\text { Nrf2 } \\
\text { (Binding } \\
\text { energy in kJ) }\end{array}$ & $\begin{array}{c}\text { NFkB } \\
\text { (Binding } \\
\text { energy in } \\
\mathrm{kJ} \text { ) }\end{array}$ \\
\hline Melatonin & -4.8 & -3.2 & -4.8 & -4.5 & -4.2 \\
\hline
\end{tabular}

As discussed earlier, melatonin is shown to modulate UPR pathways and that is most likely achieved by binding ATF6, PERK, XBP1. Activating the UPR reduces the incidence of UPR induced autophagy, promotes neuronal viability and helps in clearing the misfolded and aggregated proteins.[26]. Besides, melatonin also modulates Antioxidant and inflammatory pathways as mentioned before. It exerts its antioxidant activity by activating the Nrf2 signaling pathway which results in the upregulation of the genes concerning with the antioxidant activity.[9]. Lastly, it exerts its anti-inflammatory effect by inhibiting theNFkB pathway, which will otherwise facilitate the upregulation of pro-inflammatory cytokines and chemokines.[22]

2D protein ligand interaction profiling

Protein-Ligand interaction profiling is necessary in order to know how the ligand interacts with the given protein. This information can further pave way for the development and optimization of several lead compounds. [27]ATF6 interacts with melatonin through Lys 327 and Met 331 on chain B and Lys 327 and Met 331 on chain D.PERK interacts with melatonin through Leu 598, Cys 890, Lys 892 and Phe 943. XBP1 interacts with melatonin through Glu 121 and Lys 122 on chain B. Nrf2 interacts with Melatonin through Gly 81, Phe 83 and Tyr 334.NFkBinteracts with melatonin through Asp 300, Phe 301 and Lys 337.

\section{Lipinski Rule of 5Analysis}

Table 2

Lipinski rule of 5 analysis

\begin{tabular}{|lllllll|}
\hline Ligand & Molecular & Hydrogen bond donor & Hydrogen bond acceptor & Log P & Molar Refractivity \\
& Mass & & 3 & 1.85 & 67.23 \\
\hline Melatonin & 232 & 2 & 3 & & & \\
\hline
\end{tabular}

Lipinski rule of 5 helps in determining the drug-likeness of a chemical compound on the basis of four properties ; molecular mass, log P, number of hydrogen bond acceptors, number of hydrogen bond donors[28]. As per the rule, for a compound to be designated as orally bioactive, the molecular mass must be less than 500 , the $\log P$ ( the measure of Lipophilicity) must be less than 5 , the number of hydrogen acceptors must be less than 10 , the number of hydrogen bond donors must be less than 5, [29]. Additionally, another property taken into account for determining the drug-likeness is the molar refractivity, which should be in the range of $40-130$ for it to be classified as orally bioactive. Thus, by looking at the obtained results we can infer that melatonin adheres to all the five properties, thereby making it suitable for oral administration.

\section{ADME analysis}

Table 3

ADME properties of melatonin, BBB: Blood Brain Barrier, P-Gp: P-Glycoprotein

\begin{tabular}{|c|c|c|c|c|c|c|}
\hline Ligand & Molecular weight $(\mathrm{g} / \mathrm{mol})$ & $\begin{array}{l}\text { Water solubility } \\
\text { (Log } S \text { (ESOL) }\end{array}$ & $\begin{array}{l}\text { Lipophilicity } \\
\text { (Consensus Log } P_{\mathrm{o} / \mathrm{w}} \text { ) }\end{array}$ & Gastro-Intestinal Absorption & $\begin{array}{l}\text { P-Gp } \\
\text { substrate }\end{array}$ & BBB permeant \\
\hline Melatonin & 232.28 & -2.34, Soluble & 1.98 & High & No & Yes \\
\hline
\end{tabular}

ADME analysis help in determining the safety and efficacy of a given drug compound and is a crucial component of clinical studies. ADME data is also extremely important for a drug's regulatory approval and for its successful candidature(Li, 2001.)SwissADME server not only calculates ADME properties but a number of other descriptors like pharmacokinetic properties, drug likeness nature. [31]. Melatoninhas a molecular weight of $<500 \mathrm{~g} / \mathrm{mol}$, are highly lipophilic, can be absorbed by the GI tract, are blood brain barrier permeant and are not substrates of the PGp efflux transporter. Overall, these ADME properties point out to the fact that melatonin possesses drug-like properties appropriate for the treatment of neurodegenerative diseases.

Table 4

Drug ability of melatonin, TPSA: Total Polar Surface Area, MW: Molecular Weight, nrotb: number of

\begin{tabular}{|lllll|}
\hline \multicolumn{5}{c|}{ rotatable bonds } \\
\hline Migand & miLogP & TPSA & MW & nrotb \\
\hline
\end{tabular}

The molecular properties are in accordance to the paper published by Veberet a/ in 2002, [32]. Herein the factors influencing oral bioavailability such as polar surface area, molecular weight, rotatable bonds, are taken into consideration for estimating the same. The ideal range for log P is $>5[33$ ], TPSA is $<=140$ [34], 
number of rotatable bonds $<=10$ [32]. Melatonin adheres to all the ideal properties values, thereby once again proving that it can be administered orally as a drug.

\section{Bioactivity analysis}

Table 5

Bioactivity scores of the ligands, GPCR: G-Protein Coupled

Receptor, ICM: Ion Channel Modulator, KI: Kinase Inhibitor, NRL:

Nuclear Receptor Ligand, Pl: Protease Inhibitor, El: Enzyme

\begin{tabular}{|lllllll|}
\hline Ligand & GPCR & ICM & KI & NRL & PI & EI \\
\hline Melatonin & 0.06 & -0.09 & -0.10 & -0.51 & -0.24 & -0.01 \\
\hline
\end{tabular}

If a molecule possesses a bioactivity score of more than 0 , it is considered as highly bioactive and thus exhibits significant biological activity. Whereas a bioactivity score of -0.50 to 0.0 implies that it is moderately bioactive and a bioactivity scoreunder -0.50 indicates that the molecule shows no biological activity.[35-37]. Thus from the above observations, we can say that melatonin can only function by binding GPCR. The GPCR is the primary target for a number of hormones, ions, neurotransmitters to act upon and trigger downstream signaling. Thus, this justifies melatonin's bioactivity score being only confined to binding GPCR. [38]

\section{Conclusion}

From the above observations, it is obvious that melatonin interacts with molecules of innumerable pathways. Besides, the various analyses carried out clearly demonstrates its ability to be administered orally due to its adherence to various rules and properties. Melatonin is shown to not only fix the impaired cellular processes, but has also been shown to alleviate the symptoms of neurodegenerative disorders. Scientists can even investigate ways to enhance the production of melatonin endogenously as its production rate diminishes with old age. However, further experimental studies in disease models is required to completely understand its mode of action and its potential in treating other diseases as well.

\section{Declarations}

\section{Acknowledgement}

\section{Funding}

Authors are thankful to B.S. Abdur Rahman Institute of Science \& Technology, Chennai for providing research facilities in school of life sciences. The authors also gratefully acknowledge ASEAN University network (AUN)/Southeast Asia Engineering Education Development Network(SEED)/Japan International Cooperation Agency (JICA) SPRAC (SN042/MI.KU/2020).

\section{Conflicts of interest/Competing interests}

The authors declare that there is no conflict of interest

\section{Availability of data and material}

Data will be available on request

\section{Code availability}

Not Applicable

\section{Ethics approval}

Not Applicable

\section{Consent to participate}

Not Applicable

\section{Consent for publication}

All authors read and approved the manuscript for publication

\section{Authors' contributions}

SH conceived and designed research. FHB performed experiments. All authors wrote the manuscript. All authors read and approved the manuscript.

\section{References}

1. Przedborski, S. (2008). Neurodegeneration. Neuroimmune Pharmacology, 1918, 229-237. https://doi.org/10.1007/978-0-387-72573-4_17 
2. Przedborski, S., Vila, M., \& Jackson-Lewis, V. (2003). Neurodegeneration: What is it and where are we? Journal of Clinical Investigation, 111(1), 3-10. https://doi.org/10.1172/JCl200317522

3. Jellinger, K. A. (2010). Basic mechanisms of neurodegeneration: A critical update. Journal of Cellular and Molecular Medicine, 14(3), $457-487$. https://doi.org/10.1111/j.1582-4934.2010.01010.x

4. Basha, F. H. (2021). Cellular and molecular mechanism in neurodegeneration: Possible role of neuroprotectants, (September 2020), 1-10. https://doi.org/10.1002/cbf.3630

5. Alghamdi, B. S. (2018). The neuroprotective role of melatonin in neurological disorders. Journal of Neuroscience Research, 96(7), 1136-1149. https://doi.org/10.1002/jnr.24220

6. Fernández, A., Ordõñez, R., Reiter, R. J., González-Gallego, J., \& Mauriz, J. L. (2015). Melatonin and endoplasmic reticulum stress: Relation to autophagy and apoptosis. Journal of Pineal Research, 59(3), 292-307. https://doi.org/10.1111/jpi.12264

7. Chen, D., Zhang, T., \& Lee, T. H. (2020). Cellular mechanisms of melatonin: Insight from neurodegenerative diseases. Biomolecules, 10(8), 1-26. https://doi.org/10.3390/biom10081158

8. Davinelli, S., Maes, M., Corbi, G., Zarrelli, A., Willcox, D. C., \& Scapagnini, G. (2016). Dietary phytochemicals and neuro-inflammaging: From mechanistic insights to translational challenges. Immunity and Ageing, 13(1), 1-17. https://doi.org/10.1186/s12979-016-0070-3

9. Ahmadi, Z., \& Ashrafizadeh, M. (2020). Melatonin as a potential modulator of Nrf2. Fundamental and Clinical Pharmacology, 34(1), 11-19. https://doi.org/10.1111/fcp. 12498

10. Marsh, A. P. (2019). Molecular mechanisms of proteinopathies across neurodegenerative disease: a review. Neurological Research and Practice, 1(1), 1-7. https://doi.org/10.1186/s42466-019-0039-8

11. Chauhan, N. B., \& Mehla, J. (2015). Ameliorative Effects of Nutraceuticals in Neurological Disorders. Bioactive Nutraceuticals and Dietary Supplements in Neurological and Brain Disease: Prevention and Therapy. Elsevier Inc. https://doi.org/10.1016/B978-0-12-411462-3.00027-8

12. Xiang, C., Wang, Y., Zhang, H., \& Han, F. (2017). The role of endoplasmic reticulum stress in neurodegenerative disease. Apoptosis, 22(1), 0. https://doi.org/10.1007/s10495-016-1296-4

13. Lou, G., Palikaras, K., Lautrup, S., Scheibye-Knudsen, M., Tavernarakis, N., \& Fang, E. F. (2020). Mitophagy and Neuroprotection. Trends in Molecular Medicine, 26(1), 8-20. https://doi.org/10.1016/j.molmed.2019.07.002

14. Hetz, C., \& Saxena, S. (2017). ER stress and the unfolded protein response in neurodegeneration. Nature Reviews Neurology, 13(8), $477-491$. https://doi.org/10.1038/nrneurol.2017.99

15. Adams, C. J., Kopp, M. C., Larburu, N., Nowak, P. R., \& Ali, M. M. U. (2019). Structure and molecular mechanism of ER stress signaling by the unfolded protein response signal activator IRE1. Frontiers in Molecular Biosciences, 6(MAR), 1-12. https://doi.org/10.3389/fmolb.2019.00011

16. Scheper, W., \& Hoozemans, J. J. M. (2015). The unfolded protein response in neurodegenerative diseases: a neuropathological perspective. Acta Neuropathologica, 130(3), 315-331. https://doi.org/10.1007/s00401-015-1462-8

17. Yeganeh, B., Jäger, R., Gorman, A. M., Samali, A., \& Ghavami, S. (2015). Induction of Autophagy: Role of Endoplasmic Reticulum Stress and Unfolded Protein Response. Autophagy: Cancer, Other Pathologies, Inflammation, Immunity, Infection, and Aging, 7, 91-101. https://doi.org/10.1016/B978-0-12801043-3.00005-4

18. Markouli, M., Strepkos, D., Papavassiliou, A. G., \& Piperi, C. (2020). Targeting of endoplasmic reticulum (ER) stress in gliomas. Pharmacological Research, 157(March), 104823. https://doi.org/10.1016/j.phrs.2020.104823

19. da Silva, D. C., Valentão, P., Andrade, P. B., \& Pereira, D. M. (2020). Endoplasmic reticulum stress signaling in cancer and neurodegenerative disorders: Tools and strategies to understand its complexity. Pharmacological Research, 155(February), 104702. https://doi.org/10.1016/j.phrs.2020.104702

20. Luo, F., Sandhu, A. F., Rungratanawanich, W., Williams, G. E., Akbar, M., Zhou, S., ... Wang, X. (2020). Melatonin and autophagy in aging-related neurodegenerative diseases. International Journal of Molecular Sciences, 21(19), 1-31. https://doi.org/10.3390/ijms21197174

21. Srinivasan, V., Pandi-Perumal, S. R., Maestroni, G. J. M., Esquifino, A. I., Hardeland, R., \& Cardinali, D. P. (2005). Role of melatonin in neurodegenerative diseases. Neurotoxicity Research, 7(4), 293-318. https://doi.org/10.1007/BF03033887

22. Liu, T., Zhang, L., Joo, D., \& Sun, S. C. (2017). NF-kB signaling in inflammation. Signal Transduction and Targeted Therapy, 2(March). https://doi.org/10.1038/sigtrans.2017.23

23. Ahmed, S. M. U., Luo, L., Namani, A., Wang, X. J., \& Tang, X. (2017). Nrf2 signaling pathway: Pivotal roles in inflammation. Biochimica et Biophysica Acta Molecular Basis of Disease, 1863(2), 585-597. https://doi.org/10.1016/j.bbadis.2016.11.005 
24. Sekar, A., Soundhararajan, R., \& Srinivasan, H. (2021). In silico analysis of quercetin and its analogues against targeted proteins. Biointerface Research in Applied Chemistry, 11(5), 13695-13705. https://doi.org/10.33263/BRIAC115.1369513705

25. Meng, X. Y., Zhang, H. X., Mezei, M., \& Cui, M. (2011). Molecular docking: a powerful approach for structure-based drug discovery. Current computer-aided drug design. Current Computer Aided Drug Design, 7(2), 146-157. Retrieved from

https://www.ingentaconnect.com/content/ben/cad/2011/00000007/00000002/art00008\%0Ahttps://www.ncbi.nlm.nih.gov/pmc/articles/PMC3624763/pdf/r

26. Lindholm, D., Wootz, H., \& Korhonen, L. (2006). ER stress and neurodegenerative diseases. Cell Death and Differentiation, 13(3), 385-392.

https://doi.org/10.1038/sj.cdd.4401778

27. Salentin, S., Schreiber, S., Haupt, V. J., Adasme, M. F., \& Schroeder, M. (2015). PLIP: Fully automated protein-ligand interaction profiler. Nucleic Acids Research, 43(W1), W443-W447. https://doi.org/10.1093/nar/gkv315

28. Ã, C. A. L. (2004). Lead profiling Lead- and drug-like compounds: the rule-of-five revolution, 337-341. https://doi.org/10.1016/j.ddtec.2004.11.007

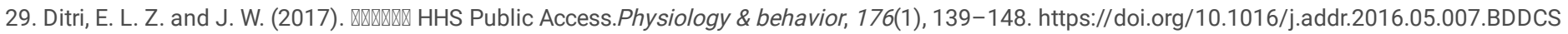

30. Li, A. P. (2001). Screening for human ADME/Tox drug properties in drug discovery. Drug Discovery Today, 6(7), 357-366. https://doi.org/10.1016/S13596446(01)01712-3

31. Daina, A., Michielin, O., \& Zoete, V. (2017). SwissADME: A free web tool to evaluate pharmacokinetics, drug-likeness and medicinal chemistry friendliness of small molecules. Scientific Reports, 7(October 2016), 1-13. https://doi.org/10.1038/srep42717

32. Veber, D. F., Johnson, S. R., Cheng, H. Y., Smith, B. R., Ward, K. W., \& Kopple, K. D. (2002). Molecular properties that influence the oral bioavailability of drug candidates. Journal of Medicinal Chemistry, 45(12), 2615-2623. https://doi.org/10.1021/jm020017n

33. Leeson, P. D., \& Springthorpe, B. (2007). The influence of drug-like concepts on decision-making in medicinal chemistry. Nature Reviews Drug Discovery, 6(11), 881-890. https://doi.org/10.1038/nrd2445

34. Lipinski, C. A. (2003). Compound Properties and Drug Quality. The Practice of Medicinal Chemistry: Second Edition (Second Edi.). Elsevier Inc. https://doi.org/10.1016/B978-012744481-9/50025-8

35. Husain, A., Ahmad, A., Khan, S. A., Asif, M., Bhutani, R., \& Al-Abbasi, F. A. (2016). Synthesis, molecular properties, toxicity and biological evaluation of some new substituted imidazolidine derivatives in search of potent anti-inflammatory agents. Saudi Pharmaceutical Journal, 24(1), 104-114.

https://doi.org/10.1016/j.jsps.2015.02.008

36. Rajasekaran, S., Prasad, P., \& Rao, G. K. (2020). iMedPub Journals Molecular Properties and Bio-Activity Score of 2 \{[ 2- ( 4-chlorophenyl ) -4- oxoquinazolin3 ( 4H ) -yl ] amino \}-N- ( substitutedphenyl ) acetamides Molinspiration software, 3, 12-14. https://doi.org/10.36648/0975-9344.12.1.153

37. Lutfiya, A. S., Priya, S., Manzoor, M. A. P., \& Hemalatha, S. (2019). Molecular docking and interactions between vascular endothelial growth factor (VEGF) receptors and phytochemicals: An in-silico study. Biocatalysis and Agricultural Biotechnology, 22(October), 101424.

https://doi.org/10.1016/j.bcab.2019.101424

38. Lenniger. (2014). Biochemsitry, 459(7245), 356-363. https://doi.org/10.1038/nature08144.The

\section{Figures}




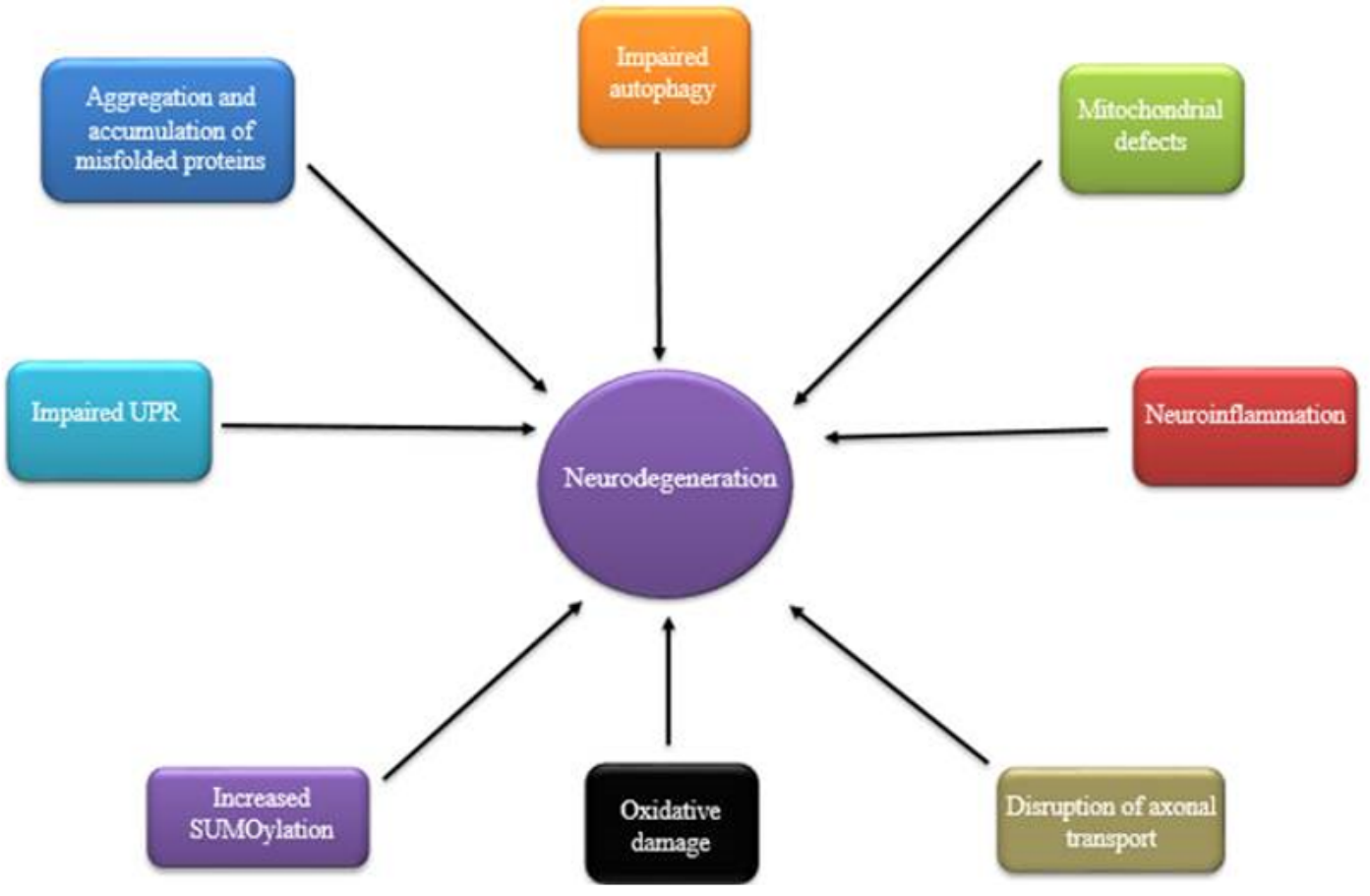

Figure 1

Various mechanisms that contribute to neurodegeneration

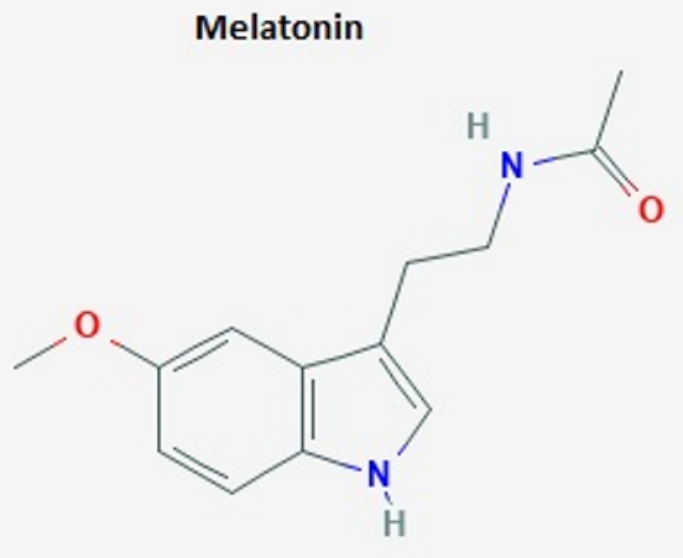

Figure 2

Structure of Melatonin 


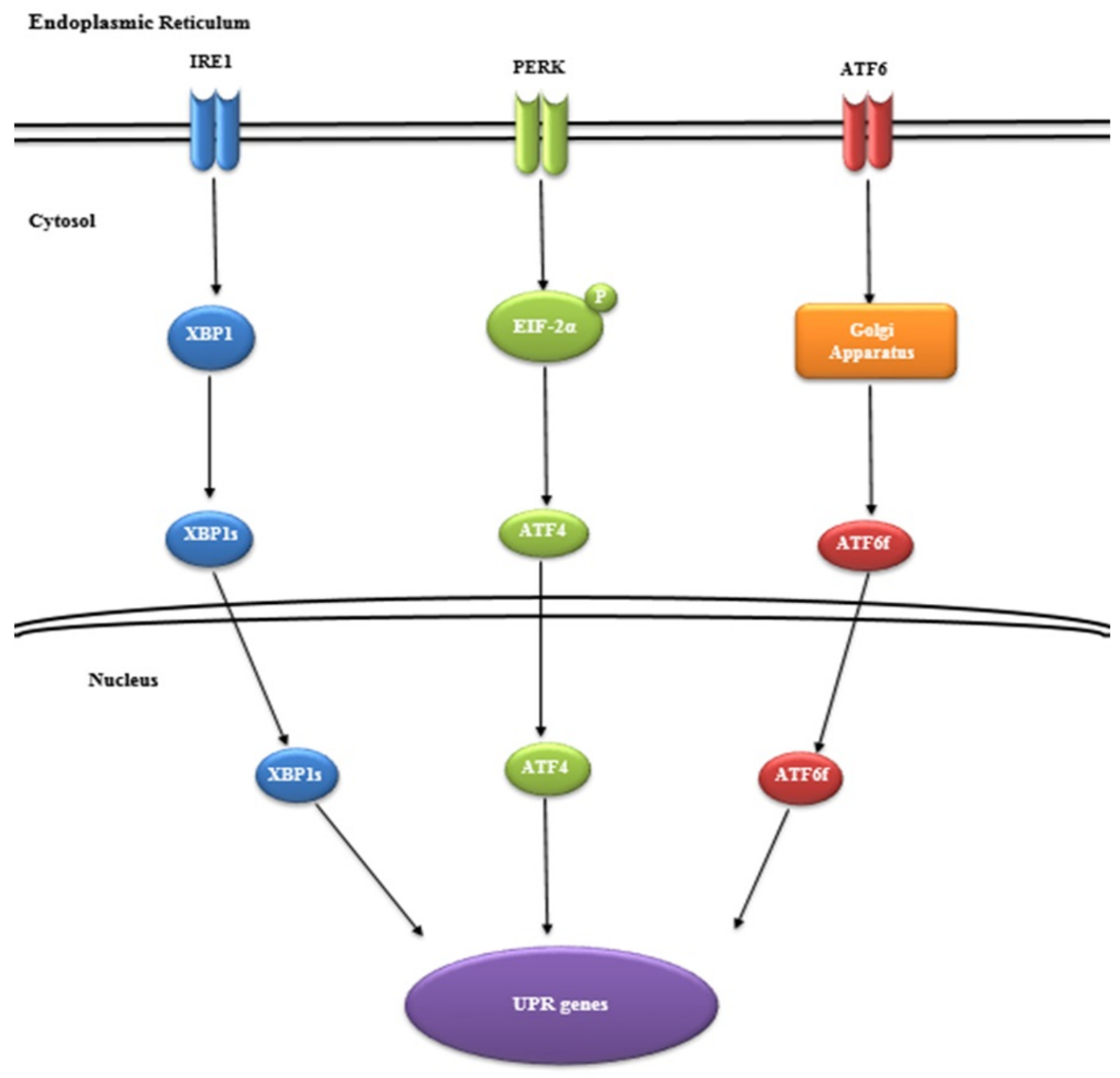

Figure 3

Diagrammatic Representation of UPR pathways 

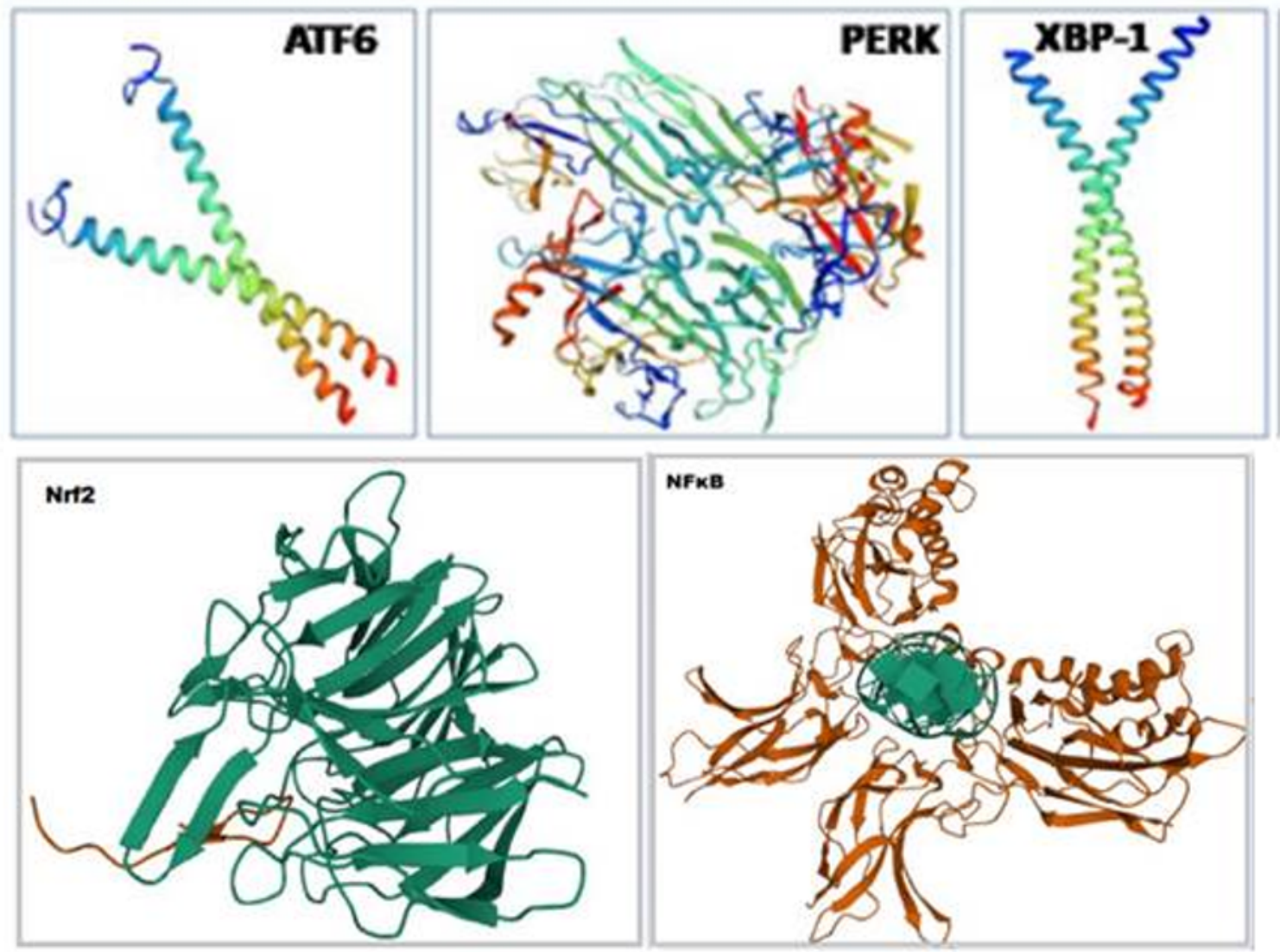

Figure 4

$3 D$ structure of the proteins 


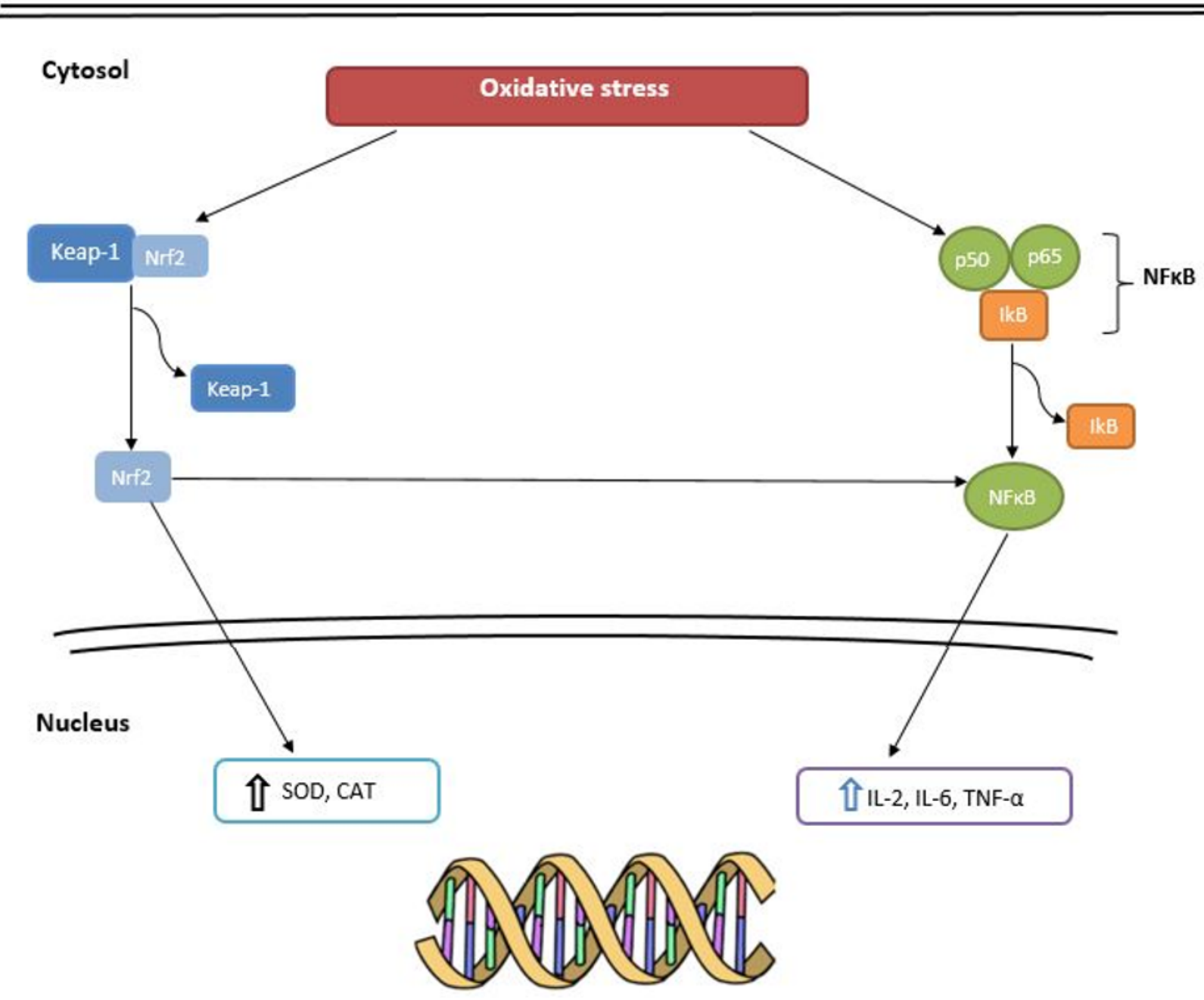

Figure 5

Upon oxidative damage, the dissociation of Keap1 and Nrf2 is facilitated, due to which Nrf2 is translocated to the nucleus and it later activates the genes controlling antioxidant defences such as Superoxide dismutase (SOD), Catalase (CAT). Under normal conditions NFKB is present as a complex of 2 subunits namely p50 and p65 and an inhibitory subunit namely $\mathrm{kBB}$. Upon appropriate stimuli, the lkB is degraded and the remaining complex is translocated to the nucleus. After reaching the nucleus, it upregulates the production of pro-inflammatory cytokinsuch as IL-2, IL-6, TNF-a. Nrf2 is also capable of interacting with NFKB and thereby inhibiting its function. 

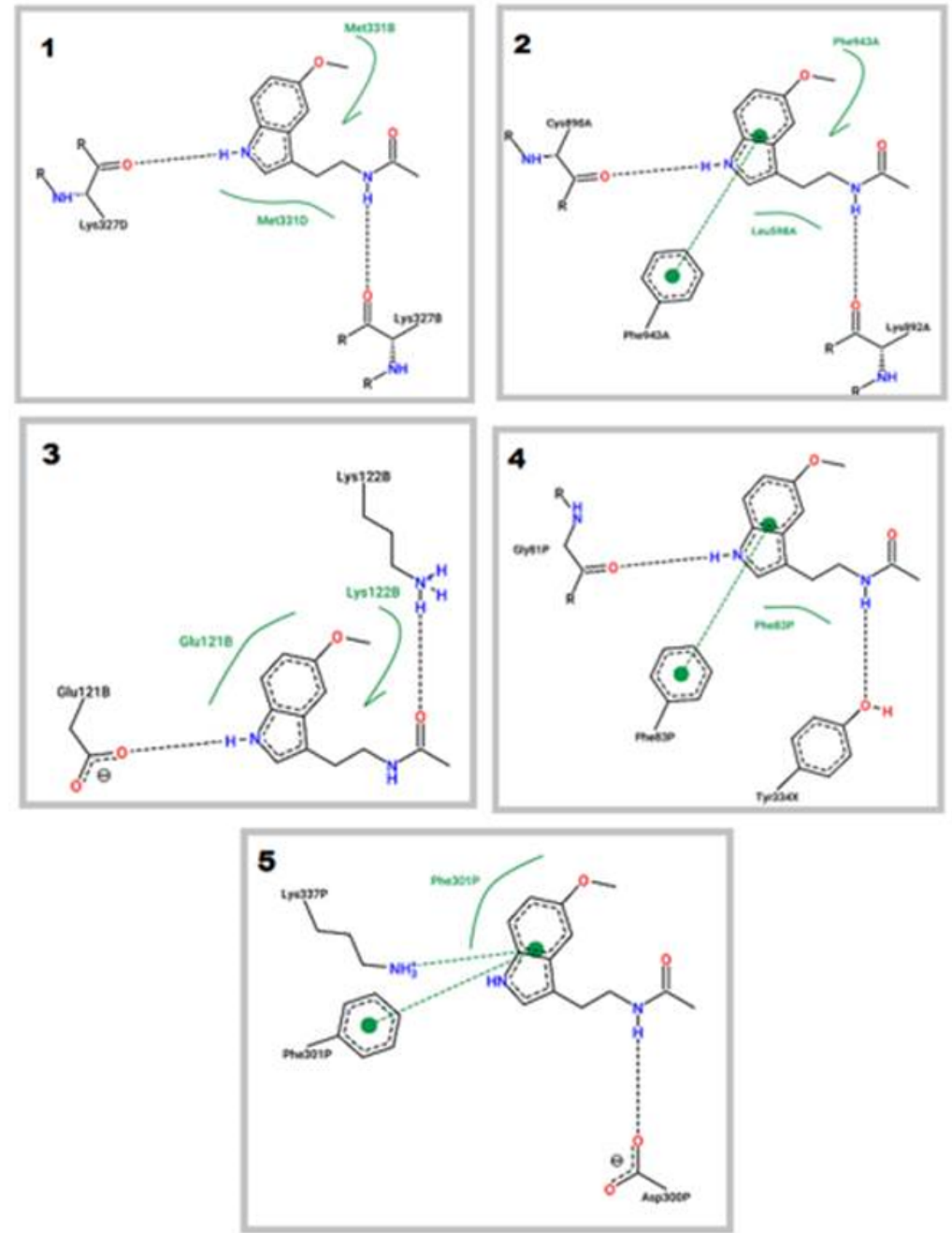

Figure 6

Interaction of melatonin with (1) ATF6 (2) PERK (3) XBP1 (4) Nrf2 (5) NFKB 